\title{
Oncogeriatrics (part 3.) \\ Influence of surgical trauma on older patients
}

\author{
Jakub Kenig \\ Department of General, Oncological and Geriatric Surgery, Jagiellonian University Medical College, Krakow, Poland
}

In the elderly, there is a unique situation where factors resulting from the ageing process and the cumulative effect of accompanying diseases interact with severe stress associated with surgery. Currently, there is no effective way to pharmacologically influence the course of pro- and anti-inflammatory responses. Therefore, surgeons should attempt to limit potential risks during procedures by conducting scheduled surgery, positioning the patient in the safe way, using minimally invasive surgical techniques, limiting intraoperative blood loss - even at the expense of prolonged surgical time, avoiding hypothermia and working in an experienced surgical and anaesthetic team. Such elements are of paramount importance for older patients undergoing surgery, particularly with frailty syndrome.

NOWOTWORY J Oncol 2019; 69, 5-6: 163-167

Key words: older patient, surgical trauma, postoperative outcomes

As mentioned previously, there are many unique aspects of perioperative care in the elderly population. During surgery there are combined effects from reduced physiological reserves (physiological aging), associated diseases (pathological aging), and an acute stress response related to surgery [1]. Published studies relating to the influence of surgical trauma in the elderly are extremely scarce. In the majority of studies elderly patients make up a part of a larger study group, with relatively few studies focussing exclusively on surgery in the elderly.

With exception to a few papers from the 1970s and 1980s, few studies have compared the neuroendocrine system, cytokine-immunological systems, and metabolic reactions in younger and older patients. The results obtained by the authors show that postoperative responses (measured by serum concentration of catecholamines, cortisol, glucose, urine nitrogen and electrolytes) are not age dependent. However, these studies used small groups of patients who had surgeries performed due to various diseases, both inflammatory and neoplastic $[2,3]$.

In the case of cytokine-immunological systems, the production of a whole range of pro-inflammatory cytokines increases postoperatively. The concentrations of interleukin-6 (IL-6) and C-reactive protein (CRP) increase in proportion to the trauma experienced and surgical technique used (e.g. laparotomy or laparoscopy). Serum IL-6 peaks approximately 18-24 $\mathrm{h}$ postoperatively. Serum CRP concentrations reaches their peak after 48-72 h. Notably, IL-6 and IL-10 levels correlate with intraoperative blood loss. The elevation of IL- 6 is significantly higher in the geriatric population. Kato and colleagues showed that the levels of IL-6 and IL-8, in response to surgical trauma in elderly patients, were significantly higher compared to a group of young adults (both after 24 and $72 \mathrm{~h}$ post-surgery). In these patients, not only an increased response, but also delayed response to the trauma was observed. However, this difference seems to be related to the type of procedure. There were no differences in IL-6 and procalcitonin levels after laparoscopic gallbladder removal between younger and older patients. The concentration of leukocytes (especially neutrophils) and platelets in the blood also increases post-surgery. Notably, there is a significant variation in the parameters mentioned above and they do not depend on the type of procedure or its extent. With age, the functioning of apoptotic pathways 
become impaired and the balance favours anti-apoptotic markers. As a result, excess immune cells may be associated with chronic inflammation [4-16].

A number of intraoperative factors influence the magnitude of acute trauma responses, including type of anaesthesia, location and extent of surgery, mode of surgery (elective, emergency), duration of surgery, intraoperative blood loss, technique of surgery, postioning on the surgical table, hypothermia, experience of surgeon and anaesthesiologist, among others [17].

Anaesthesia should be safe with smooth induction, maintenance and quick reversal without producing any cardiovascular, respiratory and nervous complications. It is currently much safer, with complications associated with anaesthesia alone are estimated to occur in $0.01 \%$ of patients. However, the impact of anaesthesia on the body of the elderly, although minimized by the development of medical technology and pharmacology, should not be neglected. Adjustment of drug selection and dosage is required for the elderly, because they are more sensitive to analgesics and sedatives; polypharmacy is also frequent. The variability in pharmacodynamics and kinetics is high. Usually, smaller doses are needed for clinical effect in comparison with the adult population, and the duration of action is prolonged [18]. The choice of anaesthesia should ensure the best possible comfort for the patient, taking into account the patient's medical history and the risks associated with it, while allowing procedures to be carried out in an efficient manner. All types of anaesthesia (general, nerve block and regional anaesthesia) are used in geriatric surgery. To date, researchers still debate the superiority of nerve block anaesthesia over general anaesthesia. Some studies emphasize that nerve block anaesthesia has less influence on the cardiovascular system, which results in fewer cardiovascular incidents, and maintenance of the respiration, leading to fewer pulmonary complications (particularly in the older patients with chronic obstructive pulmonary disease). Additionally, the use of local analgesics in nerve block anaesthesia inhibits endocrine-metabolic response by blocking hypothalamic nociceptive stimulation. However, the use of nerve block anaesthesia exposes the patient to hypothermia with all its consequences. In addition, the use of deep sedation (bispectral index <50) during nerve block anaesthesia increases the number of complications seen to the number encountered with general anaesthesia. Balanced analgesia (a combination of general and nerve block anaesthesia) seems to be the sought-after "golden mean". However, studies have not demonstrated superiority of a particular anaesthetic regime and instead recommend the anaesthetist is best placed to make this decision [19-21].

The experience of an anaesthetist may be a risk factor of postoperative complications, but this issue has been poorly investigated in older population. Cohen and colleagues analysed 11000 surgeries and found no significant correlation be- tween the anaesthetist's experience, the length of anaesthesia and postoperative mortality. Analysing complications other than death, Boettger and colleagues demonstrated a significant correlation between experience and risk of cardiological complications (three-fold increase in risk) and the necessity of postoperative prolonged ventilation (eleven-fold increase in risk). This effect was particularly pronounced when bleeding occurred during the procedure or the duration of the procedure was prolonged [22, 23].

Considering the location and extent of the procedure, thyroid or parathyroid resections are safe. There is no difference in short- and long-term results between younger and older patients (despite more serious endocrine disorder in the elderly group in the case of endocrine procedures) $[24,25]$. Treatment of the colorectal cancer in older patients is associated with a higher risk of postoperative complications and death compared to younger patients. This trend continues, depending on the publication, out to 6-18 months after surgery [26]. Subsequent results are comparable with the ones for younger people if the treatment was performed with the intention of curing the disease. In the case of gastric, pancreatic and liver surgeries, the results are significantly worse outside experienced centres [27-30]. These results can be significantly improved by pre-rehabilitation and minimally invasive surgery techniques combined with accelerated postoperative rehabilitation techniques. However, there is still a lack of good quality results for older patients with frailty syndrome.

Undoubtedly, the emergency procedure is associated with increased mortality and morbidity in older population. It results not only from the lack of possibility to optimize the patient's condition before the procedure, but also from the advanced disease process, which we often encounter due to delayed diagnosis in the elderly. Often, elderly people are unable to recall the essential elements of the interview and the medication taken. They also do not want to make their own decision, which in turn contributes to further delaying the start of effective treatment. Therefore, elderly patients should be encouraged to undergo scheduled procedures, rather than surgeries in "emergency room" conditions [31]. We have shown that laparoscopic elective cholecystectomy even in frail elderly patients is a safe procedure with acceptable postoperative complication rate [32].

Data from a small number of studies concerning the duration of surgery are contradictory [33,34]. Duration can be the result of a complex interaction of factors. The extension of the operating time depends on the complexity of the operation, possible intraoperative complications or the experience of the surgical team, but can also be the result of procedures and measures taken to prevent complications that require special attention. There are no published works describing the topic dedicated to elderly people, especially those with frailty syndrome. In our unpublished case series, the colorectal surgery duration (up to 210 minutes) was not identified as independent risk factor of the postoperative mortality and morbidity. Above 
this limit, it increased the risk of complications (particularly the delirium) in the postoperative period.

Blood transfusion significantly increases the secretion of inflammatory mediators. This is believed to be responsible for worse results in the elderly after injuries and surgery requiring transfusions. In the study by the De la Fuente and colleagues only intraoperative transfusion was the only significant intraoperative factor influencing postoperative mortality and morbidity of elderly patients undergoing pancreatoduodenectomy $(1.5295 \% \mathrm{Cl} 1.36-1.70 \mathrm{p}<0.0001$ and $1.9195 \%$ Cl 1.42-2.57 p < 0.0001, respectively) [35]. However, the authors do not agree on the volume of blood loss, which can have a negative impact. Both Mastracci and colleagues as well as Wu and colleagues declared $500 \mathrm{ml}$ of blood loss as an arbitrary value in the older population. Moreover, it seems much more justified to prolong the surgery to carry out the precise procedure with loss of blood as low as only possible $[36,37]$.

Surgical technique is one of the main factors that influence the extent of surgical trauma for both elective and emergency procedures. Many studies emphasize the advantages of laparoscopy, which is associated with reduced blood loss, less pain, faster mobilization and reduced risk of postoperative complications (particularly infection of the surgical site and pulmonary complications). Moreover, the earlier mobilization allows the elderly to return to the preoperative level of everyday life more quickly, what is essential in this age group. This could be due to the fact that the lower concentration of proinflammatory cytokines and stress reaction hormones is observed after minimally invasive procedures in comparison to standard surgical procedures [38-41].

All above-mentioned features of laparoscopy fit the principles of geriatric surgery very well. However, the role of laparoscopy in patients with serious comorbidities, where forced posture during the intraoperative period, increased intraperitoneal pressure or prolonged surgery time may have an adverse effect, remains unknown. Therefore, the decision on the surgical technique in the elderly must be made individually, especially in patients with frailty syndrome. However, the physician must be aware of the following facts $[42,43]$ :

- The surgery alone is the most important trigger of the disability and dependency in the older patients.

- Every day in bed in the postoperative period requires a week of rehabilitation.

- The first three days in bed are equivalent to $10 \%$ loss of muscles mass and ten days in bed to $1 \mathrm{~kg}$ of muscle loss.

- Three to five weeks in bed cause a $50 \%$ loss of muscle strength.

In terms of surgeon experience, several research groups observed direct correlation between surgeon and hospital volume and postoperative outcome [44-46]. However, there is still no consensus among physicians on the best way to measure the quality of treatment. At present there are three types of measures: structural (volume of the hospital and/or physician), process (check whether a physician did the right thing for the patient) and outcome measure (most direct measure, but also the hardest to get right due to high variability of patients). Each of them has some limitation and shows only the partial picture of the reality. However, there is no doubt that this factor is the key element of geriatric surgery.

The risk of peripheral nerve damage and pressure injuries from mal-positioning is significantly increased due to skin atrophy and decreased skin integrity in older population. Therefore, care should be taken to ensure proper positioning and padding of bony prominences. Possible intraoperative risk factors for pressure ulcers are as follows: intraoperative transfusion, hypothermia, long-lasting surgery, longer lasting hypotension, severe comorbidities, vasopressor use [47, 48].

Hypothermia (body temperature below $36.0^{\circ} \mathrm{C}$ ) is a common problem in the elderly. Loss of subcutaneous fat tissue and muscle mass together with slower metabolism and vascular reactivity renders the elderly especially sensitive to surrounding temperature changes with greater difficulty in maintaining normothermia. It causes an increase in the production of catecholamines, arterial pressure and contraction of the vascular bed. This promotes ischaemia and heart rhythm disorders, increases the risk of the surgical site infection, coagulopathy and reduces metabolism of medication. It is also an independent risk factor for postoperative cognitive disorders and delirium [49]. Anaesthesia itself also contributes to hypothermia, regardless of whether it is general or nerve block anaesthesia. In turn, maintaining normothermia reduces the number of cardiovascular complications by 55\% [50]. The only exception is neurosurgery, where it has been shown that lowering the temperature has a positive effect. Postoperative seizures are not common in the elderly, but their occurrence causes a 20-38\% increase in metabolism. This is a very unfavourable phenomenon in the early postoperative period, which may lead to myocardial infarction or cardiac arrhythmias. Older people $>65$ years of age are particularly vulnerable. In addition, maintaining "thermal comfort" reduces the magnitude of the perioperative trauma, as measured by pro-inflammatory cytokines [51].

\section{Intraoperative factors influencing the outcome of elderly patients}

- Type of anaesthesia (incl. experience of an anaesthetist)

- Positioning of the patient

- Location and extent of the procedure

- Emergency mode of the surgery

- Surgical technique (laparoscopy, experience of a surgeon)

- Duration of surgery

- Blood transfusion

- Hypothermia

Conflict of interest: none declared 


\author{
Jakub Kenig \\ Jagiellonian University Medical College \\ Department of General, Oncological and Geriatric Surgery \\ ul. Prądnicka 35-37 \\ 31-202 Kraków, Poland \\ e-mail:jkenig@cm-uj.krakow.pl
}

Received and accepted: 3 Nov 2019

\section{References}

1. Kenig J. Oncogeriatrics (Part 2.). Normal and pathological ageing. Nowotwory. Journal of Oncology. 2019; 69 (3-4): 146-149.

2. Watters JM, Redmond ML, Desai D et al. Effects of age and body composition on the metabolic responses to elective colon resection. Ann Surg. 1990; 212 (2): 213-220.

3. Blichert-Toft M, Christensen V, Engquist A et al. Influence of age on the endocrine-metabolic response to surgery. Ann Surg. 1979; 190 (6): 761-770.

4. Duda K, Ziętkiewicz M. Wpływ urazu na ustój. Podstawy chirurgii; red. Jacek Szmidt i Jarosław Kużdżał; tom 1, rozdział 5: 117-173.

5. Qian F, Guo X, Wang X et al. Reduced bioenergy and toll-like receptor 1 function in human polymorphonuclear leukocytes in ageing. Aging. 2014; 6 (2): 131-139.

6. Plonquet A, Bastuji-Garin S, Tahmasebi F et al. Immune risk phenotype is associated with nosocomial lung infections in elderly in-patients. Immun Ageing. 2011; 8: 8-14.

7. Kato M, Suzuki H, Murakami M et al. Elevated plasma levels of interleukin-6, interleukin-8, and granulocyte colony-stimulating factor during and after major abdominal surgery. J Clin Anesth. 1997; 9 (4): 293-238.

8. Vester $\mathrm{H}$, Huber-Lang MS, Kida $\mathrm{Q}$ et al. The immune response after fracture trauma is different in old compared to young patients. Immun Ageing. 2014; 11: 20-28.

9. Kato $\mathrm{M}, \mathrm{Honda}$ I, Suzuki $\mathrm{H}$ et al. Interleukin-10 production during and after upper abdominal surgery. J Clin Anesth. 1998; 10 (3): 184-188.

10. Gebhard F, Pfetsch H, Steinbach $G$ et al. Is interleukin 6 an early marker of injury severity following major trauma in humans? Shock. 2003; 19 (4): 299-304.

11. Pape HC, Schmidt RE, Rice J et al. Biochemical changes after trauma and skeletal surgery of the lower extremity: quantification of the operative burden. Crit Care Med. 2000; 28 (10): 3441-3448.

12. Ouyang $\mathrm{Q}$, Wagner WM, Wikby A et al. Compromised interferon gamma (IFN-gamma) production in the elderly to both acute and latent viral antigen stimulation: contribution to the immune risk phenotype? Eur Cytokine Netw. 2002; 13 (4): 392-394.

13. Kavathia N, Jain A, Walston J et al. Serum markers of apoptosis decrease with age and cancer stage. Aging. 2009; 1 (7): 652-663.

14. Warner HR. Aging and regulation of apoptosis. Curr Top Cell Regul.1997; 35: 107-121.

15. Perl M, Chung CS, Ayala A. Apoptosis. Crit Care Med. 2005; 33 (12): 526-529.

16. Tian Y, Tao T, Zhu J et al. Soluble tumor necrosis factor related apoptosis inducing ligand level as a predictor of severity of sepsis and the risk of mortality in septic patients. PLoS One. 2013; 8 (12): 82204-82212.

17. Mastalerz K, Kenig J, Skorus U. Intraoperative factors influencing postoperative outcomes in older patients undergoing abdominal surgery - narrative review. Indian Journal of Surgery. 2018; 80 (6): 609-615.

18. Strom C, Rasmussen LS. Chalanges in anaesthesia for elderly. Singapore Dental Journal. 2014; 35: 23-29.

19. Chu CC, Weng SF, Chen KT et al. Propensity score-matched comparison of postoperative adverse outcomes between geriatric patients given a general or a neuraxial anesthetic for hip surgery: a population based study. Anesthesiology. 2015; 123: 1 36-147.

20. Rodgers A, Walker N, Schug S at al. Reduction of postoperative mortality and morbidity with epidural or spinal anaesthesia: results from overview of randomised trials. BMJ. 2000 321: 1493.

21. Hausman MS Jr, Jewell ES, Engoren M. Regional versus general anesthesia in surgical patients with chronic obstructive pulmonary disease: does avoiding general anesthesia reduce the risk of postoperative complications? Anesth Analg. 2015; 120: 1405-1412.

22. Cohen MM, Duncan PG, Tate RB. Does anesthesia contribute to operative mortality? JAMA. 1988; 260 (19): 2859-2863.

23. Böttger TC, Hermeneit $S$, Müller M et al. Modifiable surgical and anesthesiologic risk factors for the development of cardiac and pulmonary complications after laparoscopic colorectal surgery. Surg Endosc. 2009; 23 (9): 2016-2025.

24. Canonico S, Pellino G, Pameggiani D at al. Thyroid surgery in the elderly: a comparative experience of 400 patients from an Italian University Hospital. Int Surg. 2014; 99 (5): 523-527.

25. Morris LF, Zelada J, Wu B et al. Parathyroid surgery in the elderly. Oncologist. 2010; 15 (12): 1273-1284.

26. Dekker C, van den Broek B, Bastiaannet E et al. Importance of the first postoperative year in the prognosis of elderly colorectal cancer patients. Ann Surg Oncol. 2011; 18 (6): 1533-1539.

27. Schlesinger-Raab A, Mihaljevic AL, Egert $S$ et al. Outcome of gastric cancer in the elderly: a population-based evaluation of the Munich Cancer Registry. Gastric Cancer. 2016; 19 (3): 713-722.

28. LiY, Wang S, Gao S et al. Laparoscopic colorectal resection versus open colorectal resection in octogenarians: a systematic review and metaanalysis of safety and efficacy. Tech Coloproctol. 2016; 20 (3): 153-162.

29. Sperti C, Moletta L, Pozza G. Pancreatic resection in very elderly patients: a critical analysis of existing evidence. World J Gastrointest Oncol. 2017; 9 (1): 30-36.

30. Okinaga $\mathrm{H}$, Yasunaga $\mathrm{H}$, Hasegawa K. Short-term outcomes following hepatectomy in elderly patients with hepatocellular carcinoma: an analysis of 10.805 septuagenarians and 2.381 octo- and nonagenarians in Japan. Liver Cancer. 2018; 7: 55-64.

31. Ozkan E, Mahir Fersahoglu M, Dulundu E et al. Factors affecting mortality and morbidity in emergency abdominal surgery in geriatric patients. Ulus Travma Acil Cerrahi Derg. 2010; 16 (5): 439-444.

32. Kenig J, Wałęga $P$, Olszewska $U$ et al. Geriatric assessment as a qualification element for elective and emergency cholecystectomy in older patients. World J Emerg Surg. 2016; 29 (11): 36-42.

33. Dexter SP, Martin IG, Marton J et al. Long operation and the risk of complications from laparoscopic cholecystectomy. Br J Surg. 1997; 84 (4): 464-466.

34. Jackson TD, Wannares JJ, Lancaster RT. Does speed matter? The impact of operative time on outcome in laparoscopic surgery. Surg Endosc. 2011; 25 (7): 2288-2295.

35. de la Fuente1 SG, Bennett KM, Pappas TN et al. Pre- and intraoperative variables affecting early outcomes in elderly patients undergoing pancreaticoduodenectomy. HPB. 2011; 13: 887-892.

36. Mastracci TM, Bhandari M, Mundi R et al. CAGS and ACS evidence based reviews in surgery. Operative blood loss, blood transfusion and 30-day mortality in older patients after major noncardiac surgery. Can J Surg. 2012; 55 (6): 426-428.

37. Wu WC, Smith TS, Henderson WG et al. Operative blood loss, blood transfusion, and 30-day mortality in older patients after major noncardiac surgery. Ann Surg. 2010; 252 (1): 11-17.

38. LiY, Wang S, Gao S et al. Laparoscopic colorectal resection versus open colorectal resection in octogenarians: a systematic review and metaanalysis of safety and efficacy. Tech Coloproctol. 2016; 20 (3): 153-162.

39. Wang JF, Zhang SZ, Zhang NY et al. Laparoscopic gastrectomy versus open gastrectomy for elderly patients with gastric cancer: a systematic review and meta-analysis. World J Surg Oncol. 2016; 31: 90-95.

40. Wadström J, Martin AL, Estok R et al. Comparison of hand-assisted laparoscopy versus open and laparoscopic techniques in urology procedures: a systematic review and meta-analysis. J Endourol. 2011; 25 (7): 1095-1104.

41. Bhave Chittawar P, Franik S, Pouwer AW et al. Minimally invasive surgical techniques versus open myomectomy for uterine fibroids. Cochrane Database Syst Rev. 2014; 21 (10): 4638-4640.

42. Cruz-Jentoft AJ, Baeyens JP, Bauer JM et al. Sarcopenia: European consensus on definition and diagnosis: Report of the European Working Group on Sarcopenia in Older People; European Working Group on Sarcopenia in Older People. Age Ageing. 2010; 39 (4): 412-423.

43. Cruz-Jentoft AJ, Landi F, Schneider SM. Prevalence of and interventions for sarcopenia in ageing adults: a systematic review. Report of the International Sarcopenia Initiative (EWGSOP and IWGS). Age Ageing. 2014; 43 (6): 748-759.

44. Mehta A, Efron DT, Canner JK et al. Effect of surgeon and hospital volume on emergency general surgery out- comes. J Am Coll Surg. 2017; 225 (5): 666-675.

45. Schmidt $C M$, Turrini O, Parikh $P$ et al. Effect of hospital volume, surgeon experience, and surgeon volume on patient outcomes after pancreaticoduodenectomy a single-institution experience. Arch Surg. 2010; 145 (7): 634-640

46. Waljee JF, Ohye RG. Surgeon experience and outcomes: an age old question. Circ Cardiovasc Qual Outcomes. 2017; 10: e003533. DOI: 10.1161/ CIRCOUTCOMES.117.003533. 
47. Aronovitch SA. Intraoperatively acquired pressure ulcer prevalence: a national study. J. Wound Ostomy Continence Nurs. 1999; 26 (3): 130-136.

48. Mohanty S, Rosenthal RA, Russell MM et al. Optimal perioperative management of the geriatric patient. Best practices guideline from ACS NSQIP®/American Geriatrics Society.

49. Shoair OA, Grasso li MP, Lahaye LA et al. Incidence and risk factors for postoperative cognitive dysfunction in older adults undergoing major noncardiac surgery: a prospective study. J Anaesthesiol Clin Pharmacol. 2015; 31: 30-36.

50. Kavanagh T, Buggy DJ. Can anaesthetic technique effect postoperative outcome? Curr Opin Anaesthesiol. 2012; 25: 185-198.

51. Fred C, Ford S, Wagner D et al. Intraoperatively acquired pressure ulcers and perioperative normothermia: a look at relationships. AORN J. $2012 ; 96$ (3): 251-260. 